\title{
OTHONOMICS
}

Revista de economía, empresa y sociedad

Dosier "Género y empresa: hacia la igualdad real»

Coordinadora: Laura Lamolla Kristiansen

ORGANIZACIONES SALUDABLES

\section{Fmpresas competitivas que cuentan con entornos inclusivos y respetuosos}

\section{Ana M. González Ramos}

Profesora asociada del Departamento de Sociología de la Universitat Autònoma de Barcelona.

\begin{abstract}
RESUMEN El hecho de que las mujeres no ocupen posiciones de responsabilidad en empresas altamente competitivas del sector tecnológico evidencia la falta de adaptación de las organizaciones a la presente realidad: las mujeres son la mitad de la población y están suficientemente preparadas para ocupar cargos profesionales, pero raramente son bienvenidas. Las explicaciones liberalistas, es decir, maximizadoras del principio de libertad como regulador del mercado, suponen que las mujeres no están porque toman decisiones privadas que implican una falta de ambición o compromiso con las empresas. Frente a esta evidencia sostengo que las organizaciones son estructuras sociales poco saludables, que imponen reglas y valores incapaces de adaptarse a las diferentes realidades de mujeres y hombres que ansían otra cultura organizativa -que acompañe mejor a sus estilos de vida-. Este trabajo aborda esta cuestión aportando evidencias sobre la base de dos ejes generadores de riesgos psicosociales en la sociedad del conocimiento: (1) el desajuste entre las vidas personales y laborales, y (2) el desajuste durante la gestión de las relaciones personales y emocionales, así como de las prácticas cotidianas en las organizaciones, en el ámbito familiar y, en general, en la sociedad. Los resultados de este trabajo muestran que las empresas tecnológicas están haciendo frente a algunos cambios relacionados con el perfil de sus trabajadores, sin embargo, requiere una reflexión más profunda y cambios culturales que abandonen la idea de la existencia de un ideal masculino de profesional.
\end{abstract}

PALABRAS CLAVE conciliación vida laboral-personal; tipos de contratación; género; ciclo vital; salud; relaciones de poder 
HEALTHY ORGANIZATIONS

\title{
Competitive companies with inclusive and respectful environments
}

\begin{abstract}
The fact that women do not occupy positions of responsibility within highly competitive companies in the technological sector demonstrates how organisations are failing to adapt to the present reality: women are half of the population and are sufficiently prepared to occupy professional posts, but they are rarely welcomed. The liberalist explanations, that is to say, those which maximise the principle of freedom as regulating the market, indicate that women are not present because they take private decisions which involve a lack of ambition or commitment to the companies. In the face of this evidence, I maintain that the organisations are less than healthy social structures, which impose rules and values that cannot be adapted to the differing realities of women and men who are eager for another organisational culture - one which could fit better with their lifestyles. This work tackles this question by providing evidence on the basis of two generative axes of psychosocial risks for the knowledge society: (1) the imbalance between personal and work lives (2) the imbalance during the management of personal and emotional relations, as well as in the daily practices in the organisations, in the family and, in general, in society. The results of this work show that the technological companies are facing up to some changes relating to the profile of their workers, however, there is a need for a more profound reflection and cultural changes which abandon the idea that there exists a male professional ideal.
\end{abstract}

KEYWORDS Work-life balance; labor arrangement types; gender; life-course cycle; health; power relationships

\section{Introducción}

Algunas de las empresas más competitivas del sector de las tecnologías de la información y las comunicaciones (TIC) son conscientes de que deben combatir las desigualdades de género para alcanzar el éxito. En primer lugar, apuestan por que las innovaciones no estén alejadas de la realidad de las mujeres ni de sus intereses como usuarias y consumidoras, por lo que tienen que responder a sus expectativas y necesidades. En segundo lugar, no contar con ellas como creadoras, diseñadoras o prosumidoras representa un riesgo alto por lo que supone ignorar su mano de obra, su talento y visión (su situated knowledge, como diría Donna Haraway, 1988). En tercer lugar, porque este sector económico arroja la capacidad de ser punta de lanza de la economía y desarrollador de las herramientas que acompañan los grandes cambios en los hábitos y estilos de vida de la sociedad. En cuarto lugar, sus empresas lideran políticas inspiradas en el compromiso con las personas empleadas, a quienes proporcionan incentivos no solo económicos, sino materiales e inmateriales (tiempo, pensiones, instalaciones de ocio y descanso en sus centros, etc.). Además, han sido pioneras en la adopción de medidas relacionadas con la flexibilidad laboral y el teletrabajo para fomentar la creatividad y la productividad de su plantilla, permitiendo el cambio hacia diferentes estilos de trabajo de sus empleados y empleadas (aunque también han puesto en marcha medidas más cuestionadas, como la congelación de óvulos para las mujeres treintañeras, lanzada por Apple y Facebook en 2014). 
Pese a este conjunto de políticas laborales amigables, supuestamente benefactoras para las mujeres, la brecha de género sigue existiendo; probablemente, porque se centran en ciertos problemas laborales, pero siguen sin abordar aspectos más importantes como la cultura de trabajo y el trabajo masculinizado y acelerado (Rosa, 2013; Wajcman y Dodd, 2017; Conesa y González, 2018; Lamolla y González Ramos, 2018) que conllevan el estrangulamiento de la vida personal, en favor del éxito y del sistema de promociones y de reconocimiento institucional (lo cual, alude a la gestión de la inclusión y las políticas de igualdad). Este artículo gira en torno a la capacidad de este sector de crear prácticas corporativas que construyan organizaciones saludables, que pongan en el centro el respeto a las personas y no el resultado de su trabajo (su plusvalía en términos marxianos u «objetivos» según el modelo de gestión empresarial contemporáneo).

El presente trabajo se articula en torno a cuatro secciones: la primera muestra el marco teórico y analítico a partir de los cuales se analizan los entornos laborales competitivos e inclusivos de las empresas del sector TIC. El segundo presenta el marco metodológico donde se desglosan las fuentes de investigación de donde proceden los resultados que se discuten en este trabajo. En el tercer apartado se formulan los resultados y, finalmente, en el cuarto, se presentan las principales conclusiones y recomendaciones para que las empresas sean entornos organizativos más saludables.

\section{Cambios en las empresas que afectan a la organización del trabajo, la vida personal y la esfera profesional}

El dinamismo de los mercados laborales y el escenario de competitividad económica son el marco a partir del cual las empresas han adoptado nuevas formas contractuales (como la uberización auspiciada por la llamada "gig economía» o «economía del click», Fernández Avilés, 2017), de gestión de la actividad productiva y nuevas relaciones laborales con los empleados (basadas en objetivos alcanzados y/o proyectos realizados a partir de los cuales generar promociones, autonomía profesional, responsabilización de los resultados, etc.). Por su parte, la ética del trabajo, y particularmente de aquellas personas con mayor cualificación, ha propiciado intensificación en las horas de trabajo, hiperexigencia en la búsqueda del éxito profesional y la aceptación de que el sistema de promoción sea diseñado autónomamente y no por parte de la empresa. Es decir, que cada persona decide su futuro profesional cambiando de una empresa a otra (por tanto, el sistema de promociones se desvincula de la responsabilidad de la organización y de la dirección de la empresa).

Por otra parte, la incorporación de las TIC en los procesos de trabajo ha aumentado la flexibilidad y la independencia de los trabajadores con el lugar de trabajo. En este punto, las empresas del sector alardean de su capacidad de innovación y de adaptación a la globalización de los mercados, puesto que la digitalización del trabajo permite deslocalizar a empleados y clientes. Además, la digitalización ha permitido agilizar los procesos productivos, la inmediatez de los contactos entre diferentes nodos cualquiera que sea su ubicación en el planeta y, al menos supuestamente, ha llegado para beneficiar a las personas empleadas. Pero, la introducción de las tecnologías en el mundo del trabajo conlleva aspectos positivos y negativos. Entre los positivos, la capacidad de autoorganización de las personas empleadas; entre los negativos, el hecho de que la conectividad genera una relación de disponibilidad total entre la empresa y sus empleados (Huws, 1984; Murillo, 2006; Eurofound, 2017; Eurofound e ILO, 2017). Tanto es así, que el derecho a la desconexión ha sido regulado por la Unión Europea (European Framework Agreement on Telework, 2002).

A todo ello hay que sumar los efectos negativos que soportan las mujeres por el hecho de que las organizaciones siguen sosteniéndose sobre culturas corporativas androcéntricas, compuestas de prácticas y valores desiguales inconscientes; en donde, además, se señala la existencia de políticas de igualdad, pero que están escasamente implementadas (se les dedican pocos recursos, motivan aún muchas resistencias; lo que les impide avanzar desde la igualdad formal a la igualdad real, y desde las políticas de conciliación a las de igualdad de oportunidades); y en donde, en general, las relaciones de poder establecidas generan más dificultades para las 
mujeres que para los hombres en el sistema de ascenso y promoción (Ackers, 1990; Connell, 2006; Wilson et al., 2006; O’Neil et al., 2008).

En relación con la salud laboral en las organizaciones empresariales contemporáneas, se han identificado diversos problemas etiquetados como riesgos psicosociales: fatiga mental, estrés, sobrecarga cognitiva, exceso de control y monitorización, supervisión y exigencia, aislamiento, acoso laboral, cyberbulling, etc. La mayoría de estos conceptos han sido catalogados como patologías, son objeto de regulación laboral y de supervisión por parte de las inspecciones del trabajo, además de discutidas en los convenios laborales de las empresas. Pero, los riesgos psicosociales para la salud laboral de los empleados del siglo XXI están sobre todo relacionados con la intensificación del trabajo, el solapamiento del tiempo destinado al descanso y cuidados con las jornadas laborales que se completan en el hogar, nuevas pautas de trabajo relacionadas con el nomadismo, internacionalización y/o globalización que afectan al ensanchamiento de las franjas de tiempo laborales para satisfacer la demanda de clientes y colaboradores que se encuentran en distintos husos horarios.

Por todo ello, la mayoría de consecuencias sobre la salud de las personas empleadas de alta cualificación se están desarrollando más rápidamente y de una manera más intensa de lo que cabría esperar, ya que:

- La mayoría de estos problemas son inconscientes para los actores; muy al contrario, se asumen como elementos intrínsecos al ejercicio profesional.

- No solo están ligados a las condiciones de trabajo de las organizaciones, sino también a los cambios en los estilos de vida de las personas.

- La operacionalización que permitiría su identificación, denuncia y resolución mediante indicadores objetivos es especialmente ardua.

- Y, por tanto, muchos de esos problemas no están contemplados en la legislación laboral ni en los protocolos de salud de atención para las empresas.

La complejidad y la relevancia de las actuales condiciones de trabajo, que conllevan importantes riesgos para la salud, requieren un marco analítico específico. En mi opinión, requieren un enfoque multidimensional que comprenda todo el ecosistema de las organizaciones empresariales y de las personas cualificadas empleadas en sectores productivos propios de la economía del conocimiento. En otro lugar, he defendido la misma idea aplicada a las instituciones de ciencia, tecnología e innovación, semejantes en cuanto a las relaciones profesionales y personales al sector de las tecnologías (González Ramos, et al., 2019). Este marco analítico pone en el centro el trabajo desarrollado profesionalmente (no salarialmente) en coordinación con la vida (personal, familiar, privada) de los profesionales altamente cualificados; se fija en sus estilos de vida y en modelos familiares y de cuidado específicos; ello requiere considerar las relaciones de poder, las desigualdades de género y las debidas a la edad o la etapa en el ciclo vital, entre otras.

El siguiente gráfico ha sido elaborado con la finalidad de ilustrar estos factores que estructuran los entornos laborales de las personas empleadas. Puesto que el trabajo se entroniza alrededor de las vidas de estas personas, hay cuatro subsistemas que lo atraviesan: vida, profesión, relaciones de desigualdad y relaciones de poder. 


\section{Figura 1. Factores que estructuran los entornos laborales de las personas empleadas}

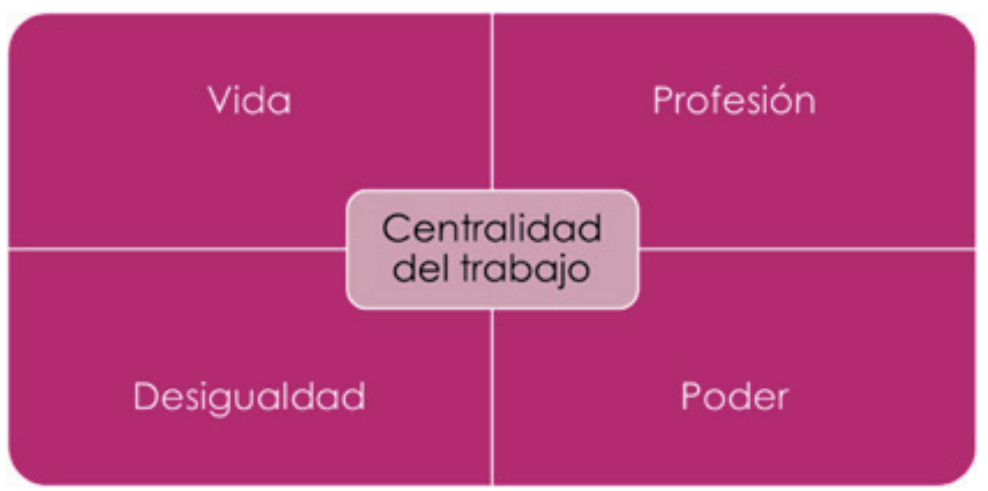

Fuente: elaboración propia.

Siguiendo el modelo anterior, propongo un marco de análisis basado en la identificación de dos ejes generadores de riesgos psicosociales debido a la tensión existente en diferentes planos del esquema anterior:

- Desajuste entre las vidas laboral y personal.

- La gestión de las relaciones personales, emocionales y las prácticas cotidianas.

El primer eje de desajuste tiene que ver con la conciliación, con la (in)adecuación de las jornadas laborales completas y parciales existentes en el mercado laboral, con las discriminaciones del mercado laboral por razón de edad o ciclo vital, y con la asunción de tareas no remuneradas ni reconocidas pero esenciales para el funcionamiento de la sociedad y/u organización. El segundo eje comprende situaciones de desigualdades vividas por las personas empleadas como miembros de una organización, por ejemplo, el acoso, el workaholismo, el burnout y la falta de armonización del esfuerzo realizado y las recompensas logradas. Todo lo cual está relacionado con un diseño androcéntrico de los entornos laborales y con la existencia de un universal masculino sobre el trabajo, el éxito y el progreso profesional (Acker, 1990; Connell, 2006; Murillo, 2006).

\section{Notas metodológicas}

El marco de análisis y resultados de este trabajo se ha desarrollado a partir de diferentes proyectos de investigación, además de la labor docente y la actividad científica como ponente en conferencias y congresos. En primer lugar, deben incluirse los proyectos «GENERA: generación de una economía del conocimiento más competitiva e inclusiva» (convocatoria Retos, Ministerio de Economía) y «GENTALENT: incorporando, reteniendo y promocionando el talento femenino en el sector de las tecnologías» (convocatoria Recercaixa, La Caixa y ACUP). El primero se centra en la situación de las personas que trabajan en universidades, centros de investigación y empresas de I+D e innovación. El segundo, en las condiciones de trabajo de las mujeres en el sector de las tecnologías. Ambos proyectos analizan la situación de los profesionales cualificados; por lo que se exploraron cuestiones tales como el tiempo de trabajo, los tipos de contratos, las situaciones de bienestar y de malestar dentro de sus centros de trabajo, el sistema de promoción y reconocimiento, así como sus expectativas y ética de trabajo. En segundo lugar, el trabajo de síntesis de los resultados de investigación, ajustándolos a la transferencia del conocimiento al alumnado de postgrado del curso «Polítiques d'Igualtat entre Homes i Dones a Europa: Treball Salut i Vida Quotidiana» de la Universitat Autònoma de Barcelona. Por último, la elaboración de la información para la difusión científica participando en varias conferencias, de entre las cuales destaco las siguientes plenarias invitadas: "XX Congreso Internacional del Instituto Universitario de Estudios Feministas y de Género Purificación Escribano. 
Salud, Emociones y Género» 2016, «XII Congreso Nacional de Ergonomía y Psicosociología, Avilés 2019», y la comunicación en el «XIII Congreso de la Federación Española de Sociología (FES)».

Los resultados se basan en información cuantitativa y cualitativa procedente de fuentes de datos primarias y secundarias. Así, en relación con la metodología cuantitativa, se han analizado datos de la Encuesta de Población Activa, del Ministerio de Educación y Ciencia, de informes de la Organización Internacional del Trabajo y Eurostat; asimismo, se han diseñado y tratado los datos procedentes de dos encuestas online de diseño propio (pueden consultarse en la web genera-uoc.com y genera-uoc.com/gentalent); y se han recopilado datos publicados de diferentes agencias de evaluación españolas, textos legales e informaciones variadas provenientes de revistas y diarios especializados. Sobre la metodología cualitativa hay que añadir que se han realizado 10 estudios de casos en diferentes centros y departamentos de la geografía española, lo cual ha supuesto examinar las normativas de promoción y contratación de cada centro, desarrollar alrededor de 90 biografías y egogramas, realizar grupos de decisión simulando concursos de contratación con currículos ficticios y, finalmente, se han entrevistado a responsables de áreas de recursos humanos de empresas, ETT, empresas de captación de talento y similares.

\section{Evidencias de cambios saludables y otros no tanto en las organizaciones del sector de las tecnologías}

Suele atribuirse a las empresas TIC que hayan sido líderes en la implementación de innovadoras medidas de gestión de las personas que trabajan en sus centros de trabajo, adaptando las relaciones laborales de sus empresas a la ética del trabajo y estilos de trabajo de los más jóvenes. Medidas como la implantación de trabajo flexible, la conciliación, el trabajo remoto y también la incorporación de incentivos económicos, fiscales y sanitarios; elementos con los que compiten con otras empresas del sector para atraer talento. Las personas que entrevistamos para el proyecto GENTALENT, como responsables de la contratación de personal para las empresas TIC, afirmaron que los jóvenes se sentían más atraídos por trabajar en empresas que mostraban un perfil dinámico, innovador y que ofrecen más incentivos individuales. Esta serie de medidas que, en un primer momento, asumieron las grandes empresas como Google, Microsoft o Apple, simbolizaban estar a la vanguardia de las empresas ubicadas en las áreas más competitivas, como Silicon Valley; pero, poco a poco, se han convertido también en un sello de identidad para las organizaciones empresariales del sector. Este año, la prensa se hacía eco de las medidas implantadas por la empresa Software Delsol, una empresa no demasiado grande ni conocida de desarrollo y mantenimiento de software de gestión para PYMES (pequeñas y medianas empresas). Aunque no es una de las empresas líderes, se alinea con ellas al poner en marcha políticas de conciliación, reducción horaria, incentivos económicos para sus trabajadores, seguro médico, comedor y gimnasio; medidas que se consideran ventajosas para sus empleados.

Sin embargo, si se observan los datos globales, ni siquiera estas medidas parecen estimular la retención del talento femenino. Según EUROSTAT, en 2018 las personas empleadas como especialistas TIC en Europa ascendían a 8,9 millones de personas: 83,5\% de hombres, respecto a tan solo el 16,5\% de mujeres. Aún peor, en diez años, en la EU-28 (a la que se refieren todos los datos), la población femenina ha descendido casi seis puntos (pues en 2008 las mujeres representaban 22,2\%). La tasa de variación anual promedio de hombres y mujeres especialistas del sector subraya esta tendencia: mientras la tasa masculina ha aumentado el 4,2\% durante el periodo 2008-2018, la de la población femenina ha experimentado el 0,5\% de crecimiento anual. Por tanto, durante esta última década, el número total de especialistas hombres aumentó el 51,6\%, mientras que el incremento del número total de especialistas TIC mujeres fue de tan solo 5,1\%. Por países, algunos países del este, como Hungría y Eslovaquia contribuyeron a que la tasa de crecimiento masculina aumentara considerablemente. En términos absolutos, los datos más positivos para las mujeres se concentraron en países como Alemania (273.000 trabajadoras), Reino Unido (265.000 trabajadoras), Francia (181.000 trabajadoras) y España (algo más de 100.000 trabajadoras). 
Por tanto, ni siquiera estas medidas, aparentemente positivas y atractivas, parecen alentar o reforzar la presencia de las mujeres en el sector TIC. ¿Cómo explicamos este desajuste? Nuestro marco de análisis ofrece pistas para esclarecer sus causas. En primer lugar, el desajuste entre el mercado laboral y las vidas de las mujeres presenta varias evidencias. La primera de ella se apunta en el informe Eurofound (2017), donde se muestra cómo la jornada de trabajo se ajusta perfectamente a las preferencias de los hombres respecto a la jornada laboral que desean tener, es decir, si quieren trabajar a tiempo completo o parcial. Sin embargo, el mercado laboral no proporciona las jornadas laborales que desean tener las mujeres, quienes trabajan mucho más o mucho menos de lo que desearían. Así, mientras la curva que representa la jornada laboral de los hombres se ajusta a una curva normal, la de las mujeres es achatada y más abierta en los extremos. También la EPA abunda en este desajuste cuando contabiliza el ajuste del trabajo desarrollado por hombres y mujeres con sus preferencias horarias. Las mujeres empleadas en empresas TIC parecen más dispuestas que los hombres a trabajar menos horas incluso si recibieran un salario menor (González et al., 2017). En nuestro trabajo (González et al., 2017; Lamolla y González, 2018) hemos atribuido este resultado a un modo diferente de entender el trabajo y el uso del tiempo de hombres y mujeres, relacionado con sus roles de género y la necesidad de gestionar distintas esferas de la vida privada, profesional y familiar.

Una segunda evidencia se refiere a los desajustes del mercado laboral respecto a las mujeres atendiendo a su ciclo vital. También a partir de datos de la EPA para la población empleada en el sector TIC (González et al., 2017), se apunta al hecho de que la tasa de empleo más alta en las mujeres jóvenes (16-24 años) y de la población femenina con más edad (46-64 años) respecto a los mismos grupos de edad entre los hombres, podría deberse a prácticas de contratación discriminatorias que denotan un trato desigual hacia las mujeres. Por una parte, la brecha de género en la población desempleada en la primera etapa de la carrera profesional, es decir, de incorporación al mercado laboral, sugiere desconfianza en las mujeres jóvenes en mayor medida que entre los hombres de la misma edad. Respecto a las mujeres mayores de 45 años sugiere un cuestionamiento en su capacidad para ostentar cargos de responsabilidad propias de su etapa profesional por las cuales sufrirían una mayor tasa de desempleo que los hombres.

En la encuesta que diseñamos para el proyecto GENTALENT preguntamos a las mujeres que trabajan en el sector TIC si habían interrumpido su trayectoria laboral en algún momento, es decir, si habían estado en situaciones de desempleo o inactividad. Como cabría esperar, puesto que el sector de las tecnologías es estable y ha demostrado suficiente resistencia a los periodos de crisis económica (González Ramos et al., 2017), solo una de cada tres mujeres había experimentado esta situación; la mayoría de ellas, además, había sido una situación voluntaria y solo el 14\% se había visto impedida de seguir trabajando de manera involuntaria. Según este grupo de mujeres, los factores que habían retrasado su incorporación al mercado de trabajo habían sido por este orden: la falta de ofertas laborales, la edad y el hecho de ser mujer.

En otro estudio, basado en el mismo conjunto de datos de la encuesta GENTALENT, (Lamolla y González Ramos, 2018) se observa que las mujeres empleadas en el sector TIC afirman contar con las competencias identificadas como importantes por las empresas, que ellas las han ido adquiriendo a lo largo de su trayectoria profesional por propia experiencia y por haberse ocupado de adquirirlas mediante programas de formación específicas. En cambio, los datos indican que no son contratadas ni promocionadas en la misma medida que los hombres, lo cual denota una falta de reconocimiento a su esfuerzo. La falta de reconocimiento del esfuerzo realizado está recogida dentro del conjunto de factores que componen el segundo eje de desajuste promotor de riesgos psicosociales relacionados con la gestión de las relaciones personales y emocionales y las prácticas empresariales.

También lo son todas aquellas prácticas de contratación y de promoción denominadas "ciegas al género» que, aparentemente, son independientes del hecho de que la persona candidata sea hombre o mujer, pero que, sin embargo, ignoran las situaciones diferentes a las que se enfrenta cada grupo, los obstáculos de uso del tiempo, de gestión de redes sociales, de prácticas organizativas que lastran a las mujeres en mayor medida que a los hombres. Así, las empresas del sector, altamente masculinizadas, suelen convocar reuniones que se prolongan en el tiempo o que, precisamente para estrechar las redes de confianza entre clientes, colegas y superiores, se programan fuera de los horarios de oficina e incluso en lugares donde las mujeres suelen ser poco bienvenidas. 
Según nuestro modelo analítico, las dinámicas internas de las organizaciones empresariales pueden provocar malestares en aquellas personas que se ajustan en menor medida al ideal de trabajadores característicos de ese tipo de organizaciones (Hatmaker, 2013; Faulkner, 2014). Precisamente, las mujeres de la encuesta GENTALENT afirmaron que los aspectos de su trabajo que les producen más insatisfacción se relacionan con la falta de capacidad de influencia en las decisiones de la organización (43,3\%), no poder disfrutar de tiempo libre (tomarse un respiro) entre proyectos $(29,2 \%)$, no disponer de tiempo suficiente para completar las tareas que se les han asignado $(24,6 \%)$ y no ser consultadas sobre los objetivos de las tareas que deben desarrollar (23,3\%). Todas estas cuestiones están asociadas a la gestión de las relaciones personales y emocionales y las prácticas organizativas que las convierten en organizaciones poco saludables. El siguiente gráfico expone la batería de situaciones que se les proponía.

\section{Figura 2. Satisfacción con el trabajo desarrollado}

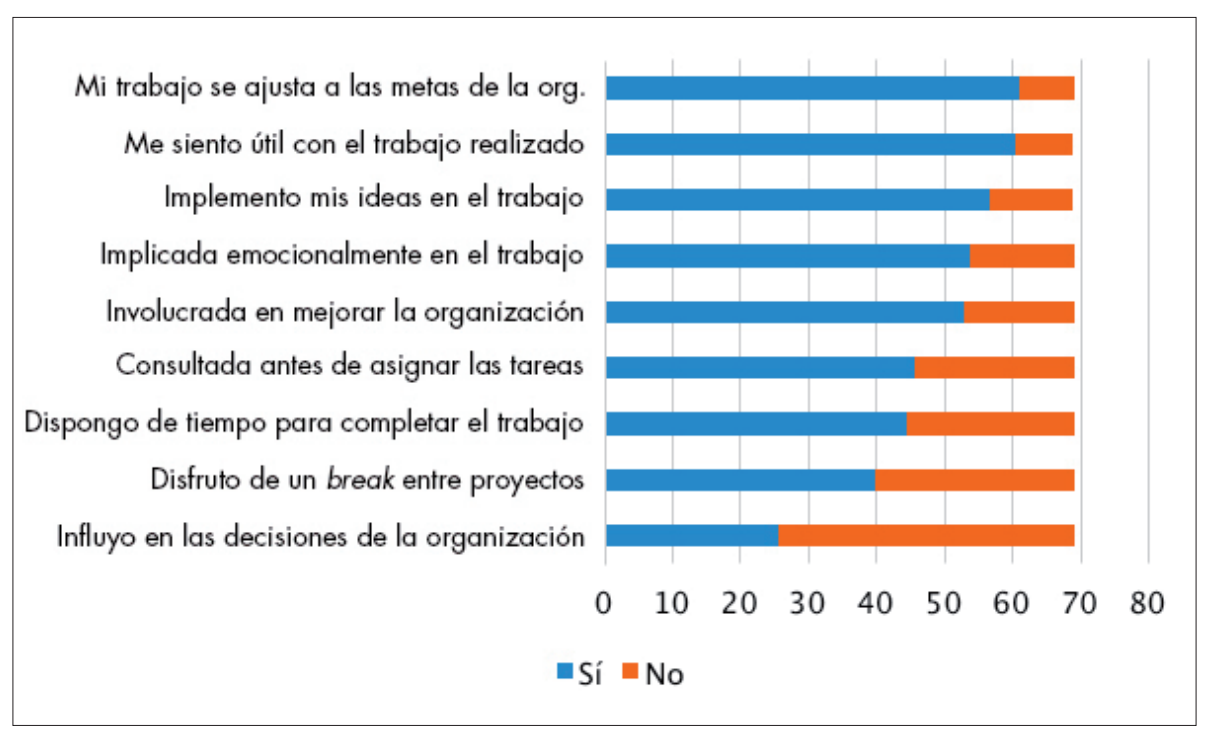

Fuente: elaboración propia, encuesta GENTALENT, 2016.

Nota: sí es el resultado del sumatorio de las categorías "siempre» y «la mayoría de las veces»; no: "raramente» y «nunca».

En este conjunto de categorías también se incluyen los síntomas de riesgos psicosociales y las relaciones laborales nocivas relacionadas con el acoso, el estrés y el burnout. Su identificación y reglamentación permite la defensa de los trabajadores, pero generalmente son reconocidas individualmente, y muy raramente de manera colectiva, como sostengo que sucede en las empresas poco saludables. Una de las pocas excepciones ha sido protagonizada por la cúpula directiva de la empresa France Télécom, cuya culpabilidad ha sido declarada por la justicia francesa en diciembre de 2019. La compañía, junto a sus excargos, ha sido declarada culpable de acoso moral a sus empleados como resultado de la nefasta gestión llevada a cabo para reestructurar la empresa en 2007. Siete exgerentes de la empresa han sido sentenciados por ser los responsables e impulsores de una cultura empresarial agresiva, poco respetuosa con su plantilla de trabajadores. La sentencia se refiere a $39 \mathrm{em}$ pleados afectados, los sindicatos añadirían el suicidio de al menos 19 personas, 12 tentativas de suicidio y otros tantos que mostraron síntomas de depresión. A pesar de los hechos, las condenas emitidas son inferiores a las máximas establecidas legalmente en la actualidad, puesto que, según la presidenta del tribunal, la legislación es insuficientemente represiva. Según la declaración de culpabilidad, no se condena la estrategia empresarial, sino los métodos empleados, lo que a mi parecer supone seguir centrándose en prácticas abusivas de personas sin atender a métodos de gestión asumidos por una gran parte de la cultura empresarial del sector. En definitiva, con esta sentencia de culpabilidad tampoco se envía un mensaje claro que haga desistir a las empresas de desarrollar estrategias agresivas de trabajo y organización. 


\section{Conclusiones}

En este trabajo se analiza la situación de las personas empleadas en empresas del sector TIC atendiendo a los cambios organizativos, las relaciones laborales y los estilos de vida de las personas altamente cualificadas. Por una parte, se abordan las condiciones laborales, por otra, se añade al análisis una perspectiva de género que trata de contribuir a esclarecer los motivos por los que las mujeres siguen siendo minoritarias en estas organizaciones. De este modo, se trata de contribuir a que las empresas puedan poner en marcha modelos de trabajo saludables tanto para los hombres como para las mujeres, pues el modelo pone en el centro a las personas y los ejes que atraviesan sus vidas personales, profesionales, laborales y familiares.

Para ello, se utiliza un modelo de análisis basado en evidencias procedentes de varios proyectos con los cuales se ha construido un marco de identificación de factores de riesgo para las personas altamente cualificadas, empleadas en ocupaciones propias de la economía del conocimiento. Este modelo de análisis refleja la existencia de desajustes debido al desajuste entre las vidas personales y laborales, así como en los problemas suscitados por las prácticas cotidianas en las empresas, en la gestión de las relaciones laborales, personales y emocionales, tanto en las empresas como en el ámbito familiar y social (aunque sobre estas dos esferas no se ha profundizado, ya que pertenecen a otro foco de atención diferente al objetivo de esta publicación).

Los resultados de este trabajo muestran que las empresas tecnológicas están haciendo frente a algunos cambios relacionados con el perfil de sus trabajadores: jóvenes, dinámicos y fuertemente orientados a la internacionalización, la competitividad y el riesgo. Sin embargo, dichas empresas proponen medidas en su mayor parte inspiradas por un ideal masculino de profesional (dado que la mayoría siguen siendo hombres y que la mayoría de las mujeres deben ajustarse a este patrón) y un estilo de trabajo que denominamos acelerado (Rosa, 2013; Conesa y González, 2018). En este sentido, he tratado de mostrar que tanto hombres como mujeres se encuentran igualmente amenazados. Aunque seguramente esta problemática se identifica en mayor medida con las mujeres, perpetuando así un estereotipo negativo sobre las mujeres, quienes serían las únicas víctimas de este modelo de trabajo. Las mujeres quizá son el caso más claro pero no exclusivamente; por ejemplo, lo acaecido en France Télécom afectó tanto a hombres como a mujeres. De todos modos, la intensidad con la que algunos problemas se avienen contra las mujeres hace más imperioso proponer una cultura de trabajo que empatice con los roles de género que las mujeres siguen desempeñando en la sociedad y, por consiguiente, en las empresas, para superar impedimentos relacionados con la desigualdad.

Entre las propuestas se incluyen políticas claras de derecho a la desconexión (digital) y al descanso (del trabajo, de las excesivas responsabilidades, etc.), la insistencia en que el trabajo autónomo no es sinónimo de trabajo aislado, el establecimiento de un sistema de recompensas relacionados no solo con los objetivos, sino con el esfuerzo invertido para conseguirlos, la erradicación de prácticas discriminatorias y/o que conducen a un trato desigual y carente de medidas que permitan la igualdad de oportunidades. En definitiva, como he apuntado al comienzo de este trabajo, que vaya más allá de las políticas de conciliación y de incentivación individual para avanzar hacia las políticas de oportunidades y de igualdad responsables con los y las trabajadoras. Recomendaciones que tienen que ver con la necesidad de que las empresas, como instituciones sociales, construyan entornos organizativos saludables.

\section{Bibliografía}

ACKER, J. (1990). «Hierarchies, jobs, bodies: a theory of gendered organizations». Gender \& Society. Núm. 4, pág. 139-158. https://doi.org/10.1177/089124390004002002.

CONNELL, R. (2006). «Glass Ceilings or Gendered Institutions? Mapping the Gender Regimes of Public Sector Worksites». Public Adminatrion Review. Núm. 66, pág. 837-849. https://doi.org/10.1111/j.15406210.2006.00652.x. 
CONESA, E.; GONZÁLEZ RAMOS, A.M. (2018). «Accelerated Researchers: Psychosocial Risks in Gendered Institutions in Academia». Frontiers of Psychology, 19 July 2018 [en línea]. https://doi.org/10.3389/ fpsyg.2018.01077.

EUROFOUND (2017). Working time patterns for sustainable work. Luxembourg: Publications Office of the European Union.

EUROFOUND/ILO (2017). Working anytime, anywhere: The effects on the world of work. Geneva and Luxembourg: Publications Office of the European Union and the International Labour Office.

FAULKNER, W. (2014). «Can Women Engineers be 'Real Engineers' and 'Real Women'? Gender In/Authenticity in Engineering”. En: WALTRAUD, E.; HORWATH, I., Gender in Science and Technology. Interdisciplinary Approaches. Bielefeld: Transcript Verlag. https://doi.org/10.14361/transcript.9783839424346.187.

FERNÁNDEZ AVILÉS, J.M. (2017). «NTIC y riesgos psicosociales en el trabajo: estado de situación y propuestas de mejora». Diritto della Sicurezza sul Lavoro. Núm. 2, pág. 69-101.

GONZÁLEZ RAMOS, A.M.; VERGÉS, N.; MARTíNEZ, J.S. (2017). «Las mujeres en el mercado de trabajo de las tecnologías». REIS. Núm. 159, pág. 61-78. https://doi.org/10.5477/cis/reis.159.73.

GONZÁLEZ RAMOS, A.M.; CONESA, E.; PONS, O.; RÄTHZEL, N. (2019). «Retos de futuro para el personal de ciencia, tecnología e innovación». XIII Congreso de la Federación Española de Sociología, Valencia. Disponible en: http://www.researchgate.net/publication/338480044_Retos_de_futuro_para_el_perso-nal_de_ciencia_tecnologia_e_innovacion?authorNameChanges\%5BAU\%3A2120203870\%5D=\&selection\%5BAU\%3A2 120203870\%5D=unknownAuthor [consultado el 13 de enero de2020].

HARAWAY, D. (1988). «Situated Knowledges: The Science Question in Feminism and the Privilege of Partial Perspective». Feminist Studies. Vol. 14, núm. 3, pág. 575-599. https://doi.org/10.2307/3178066.

HATMAKER, D. M. (2013). «Engineering identity: Gender and professional identity negotiation among women engineers». Gender, Work and Organisation. Vol. 20, núm. 4, pág. 382-396. https://doi.org/10.1111/j.14680432.2012.00589.x.

HUWS, U. (1984). The new homeworkers: new technology and the changing location of whitecollar work. London: Low Pay Unit.

LAMOLLA, L.; GONZÁLEZ RAMOS, A.M. (2018). «Tick-tock sounds different for women working in IT áreas». Community, Work \& Family. Pág. 1-16. https://doi.org/10.1080/13668803.2018.1483321.

LAMOLLA, L.; GONZÁLEZ RAMOS, A.M. (2019). «Mujeres en el sector de las tecnologías. ¿Cuestión de competencias?». Revista Española de Sociología. Vol. 28, núm. 3 (2), pág. 79-98. https://doi.org/10.22325/fes/ res.2019.39.

MURILLO, S. (2006). El mito de la vida privada. De la entrega al tiempo propio. Madrid: S.XXI.

O'NEIL, D.; HOPKINS, M.; BILIMORIA, D. (2008). «Women's Careers at the Start of the 21st Century: Patterns and Paradoxes». Journal of Business Ethics. Núm. 80, pág. 727-743. https://doi.org/10.1007/s10551-0079465-6.

ROSA, H. (2013). Social Acceleration. A New Theory of Modernity. New York: Columbia University Press.

WACJMAN, J.; DODD, N. (eds.) (2017). The Sociology of Speed. Digital, Organizational, and Social Temporalities. New York: Oxford University Press.

WILSON KOVACS, D.; RYAN, M.; HASLAM, A. (2006). "The glass cliff: women's career paths in the UK private IT sector». Equal Opportunities International. Vol. 25, núm. 8, pág. 674-687. https://doi. org/10.1108/02610150610719137.

Cita recomendada: GONZÁLEZ RAMOS, Ana. Organizaciones saludables. Empresas competitivas que cuentan con entornos inclusivos y respetuosos. Oikonomics [en línea]. Mayo 2020, no. 13, pp. 1-11. ISSN: 2339-9546. DOI: https://doi.org/10.7238/o.n13.2005 


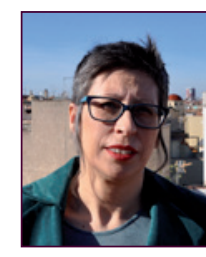

\section{Ana M. González Ramos}

AnaMaria.Gonzalez.Ramos@uab.cat

Profesora asociada del Departamento de Sociología de la Universitat

Autònoma de Barcelona

Licenciada en sociología por la Universidad de Granada en 1994 y doctora por la Universidad de Cádiz en 2004, Departamento de Estadística e Investigación Operativa. Ha sido profesora en la Universidad de La Laguna (2002-2007), en la Universidad de Cádiz (19952002) e investigadora sénior en el Internet Interdisciplinary Institute (IN3) de la UOC (2007-2019), donde dirigió el grupo GENTIC: Relaciones de Género y las Tecnologías de la Información y la Comunicación, grupo consolidado de la Generalitat (2014-2017). Actualmente es profesora asociada en la Universitat Autònoma de Barcelona.

Los textos publicados en esta revista están sujetos -si no se indica lo contrario- a una licencia de Reconocimiento 4.0 Internacional de Creative Commons. Puede copiarlos, distribuirlos, comunicarlos públicamente, hacer obras derivadas siempre que reconozca los créditos de las obras (autoría, nombre de la revista, institución editora) de la manera especificada por los autores o por la revista. La licencia completa se puede consultar en https://creativecommons.org/licenses/by/4.0/deed.es_ES.

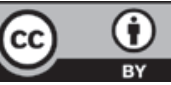

